\title{
Optimal Control Approach of Discrete Mathematical Modeling of the Spread of Gaming Disorder in Morocco and Cost-Effectiveness Analysis
}

\author{
Driss Kada $\left(\mathbb{D},{ }^{1}\right.$ Bouchaib Khajji $\left(\mathbb{D},{ }^{2}\right.$ Omar Balatif $\left(\mathbb{D},{ }^{3}\right.$ Mostafa Rachik $\left(\mathbb{D},{ }^{2}\right.$ \\ and El Houssine Labriji (iD ${ }^{1}$ \\ ${ }^{1}$ Laboratory of Information Technology and Modelling, Department of Mathematics and Computer Science, \\ Faculty of Sciences Ben M'Sik, Hassan II University, Casablanca, Morocco \\ ${ }^{2}$ Laboratory of Analysis Modeling and Simulation, Department of Mathematics and Computer Science, \\ Faculty of Sciences Ben M'Sik, Hassan II University, Casablanca, Morocco \\ ${ }^{3}$ Laboratory of Dynamical Systems, Department of Mathematics, Faculty of Sciences El Jadida, Chouaib Doukkali University, \\ Mathematical Engineering Team (INMA), El Jadida, Morocco
}

Correspondence should be addressed to Driss Kada; kadadriss.2022@gmail.com

Received 1 March 2021; Revised 26 May 2021; Accepted 10 June 2021; Published 19 June 2021

Academic Editor: Manuel De la Sen

Copyright (c) 2021 Driss Kada et al. This is an open access article distributed under the Creative Commons Attribution License, which permits unrestricted use, distribution, and reproduction in any medium, provided the original work is properly cited.

We highlight and study in this paper the phenomenon of the spread of addiction to electronic games, where the addict goes through stages before reaching the degree of addiction. In order to model this phenomenon, we have divided people into four groups, which are potential gamers, engaged gamers, addicted gamers, and gamers who have recovered from addiction. We propose a discrete mathematical model with control strategies using three controls that represent, respectively, $u_{k}$, which represents awareness of the dangers of electronic games through written and visual media; $v_{k}$, which represents the effort to direct children and adolescents to educational and entertaining alternative means; and $w_{k}$, which represents creating rehabilitation centers for addicts to quit electronic game addiction. To characterize optimal controls, we use Pontryagin's maximum principle and the system of optimality solved by an iterative method. Finally, numerical simulations are presented with and without controls. Using a cost-effectiveness analysis, we will show that the control that represents the creation of rehabilitation centers for gaming addicts is the most cost-effective strategy to control the spread of gaming addiction.

\section{Introduction}

Electronic games are seen as a traded technological commodity because they are part of modern digital culture, which affects people in different ways. Electronic games can be a big problem if they are used by the child or adolescent in a very frequent way; they can become a reason for neglecting personal, family, or educational responsibilities. Electronic games can become addictive and trying to prohibit the child or teenager from using these games will make them either sad or angry and they may want to spend more time playing these games. It should be noted that problems with electronic games are very common. The statistics about gaming addiction increased with the increase of the distribution of these games and their connection to the Internet [1]. The problem of addiction to electronic games has been classified as one of the major problems that can lead to the deterioration of public health. The concept of electronic games has been linked to many health issues such as obesity, laziness, convergence, and social illnesses. They also affect physical and mental health due to the frequent use of these games. They negatively impact children's behaviour, as shown in a study that was conducted on a group of children aged 8 to 18 over a period of three years. The results of this study indicated that violent electronic games stimulate aggressive behaviour [2]. Although children constitute the large number of people affected by watching violence, Dr. Douglas Gentili, an expert on violence in video games and doctor of evolutionary psychology at Iowa State 
University, says that electronic games affect everybody, whether they are aggressive in nature or not [3]. The World Health Organization has officially classified (International Classification of Diseases ICD-11; WHO, 2018) continuing to play video or electronic games as an addiction leading to mania and has announced that people with this mania have certain characteristics like the inability to stop gaming while winning. According to the organization, a person is classified as having this disorder if their addictive behaviour persists for 12 months. However, the diagnosis can be confirmed in a shorter period of time if it is certain that all symptoms are present. The warning about the severity of electronic gaming addiction is not new, but the formal classification of this addiction as a pathological obsession by the World Health Organization can be a major impetus to raise awareness of this disorder and take serious action in families and societies to counter it [4]. For years, research has been going on to study and understand the effect of electronic games on the behaviour and health of children. In 2000, Funk et al. [5] examined the relationships among time commitment, preference for violent games, and self-concept in 364 fourth and fifth graders. Funk et al. [6] examined correlations between the preference for violent electronic gaming and adolescents' self-perception of emotional behaviours and feelings. In 2020, Etindele Sosso et al. [7] investigated the prevalence of insomnia, excessive daytime sleepiness, anxiety, and depression among African gamers and also described epidemics of gaming disorder in the nine African countries, including Morocco, where the prevalence of addiction was described on electronic games, the average number of hours of gaming per week, the period during which the participant considers him/herself to be a gamer, and the type of device used for gaming along with age and gender (see Figure 1).

Mathematical modeling and control theory are considered as two of the most necessary tools to represent, simulate, and control the evolution of some phenomena including ecological, social, and economic ones. These tools help convert the phenomenon into mathematical equations and also to formulate study, analyze, and interpret their results. For example, Brida and Cayssials [8] generalize the classical model of determination of production prices for two commodities by introducing dynamics generated by the possibility that the port rate can be computed using prices of different stages. Wang and Petrosian [9] consider and describe the class of cooperative differential games with nontransferable utility and the process of construction of the optimal Pareto strategy with continuous updating. Guo and $\mathrm{Li}$ [10] establish a new online game addiction model with low- and high-risk exposure and use the optimal control theory to study the optimal solution problem with three kinds of control measures (isolation, education, and treatment). The work by Akanni et al. [11] formulated and analyzed a mathematical model for population dynamics of financial crime with optimal control measures. Kouidere et al. [12] studied an optimal control approach of mathematical modeling with multiple delays of the negative impact of delays in applying preventive precautions on the spread of the COVID-19 pandemic with a case study of Brazil.
Other models from optimal control problems and population dynamics can be found in [13-20].

In this research, we will adopt discrete-time modeling where statistical data are collected at a discrete time (day, week, month, and year). Therefore, it is more direct, more convenient, and more accurate to describe a phenomenon using discrete-time modeling compared to continuous-time modeling. Also, we mainly shed light on the category of addicts and make a differentiation between practitioners and addicts of electronic games. Eventually, we propose strategies for optimal control of the spread of this addiction. We add the cost-effectiveness ratio (ICER) to be able to choose between strategies based on obtaining better results at a lower cost.

In this work, we propose a new model that describes the dynamics of electronic game addiction. The population that we study consists of children and adolescents aged less than 24 years old within Morocco. The population under study is divided into four compartments, which are potential gamers, engaged gamers, addicted gamers, and recovered gamers. Our main objective is to propose an optimal control strategy that will minimize the number of addicted gamers. To achieve this objective, we adopt three controls that represent awareness through media, guidance on alternative educational and recreational methods, and creating rehabilitation centers for electronic game addiction.

This paper is organized as follows. In Section 2, we propose a PEAR mathematical model that describes the dynamics of a population that reacts to the spread of the E-game infection. In Section 3, we present an optimal control problem for the proposed model where we give some results concerning the existence of the optimal control, and we characterize the optimal controls using the Pontryagin maximum principle in discrete time. Numerical simulations through MATLAB software and the cost-effectiveness analysis are given in Section 4. Finally, we conclude the paper in Section 5.

\section{Mathematical Model and Numerical Simulation of Gaming Disorder}

2.1. Description of the Model. We consider a discrete mathematical model PEAR that describes the dynamics of a population having gaming disorder. We divide the population into four compartments. The following illustration will show disease trends in the compartments in Figure 2.

2.1.1. The Potential Gamers P. It represents children and youth who are vulnerable to infection or who are more likely to become addicted to electronic games. This compartment is increased by the recruitment rate denoted by $\Lambda$ and the risk factor for transmission from recovered persons to potential gamers $\gamma_{3}$. It is decreased by the rate $\alpha_{1}$ coefficient of transmission from potential persons to engaged gamers due to the effects of advertising for electronic games through media. Also, it is decreased by an effective contact with engaged gamers at rate $\beta_{1}$ (the rate of patients who become engaged gamers because of the negative contact with the 


\begin{tabular}{|c|c|c|c|c|c|c|c|c|c|c|}
\hline Country & Total & Age & Men & Women & $\begin{array}{l}\text { Mean hours } \\
\text { of gaming/ } \\
\text { week }\end{array}$ & $\begin{array}{l}\text { Mean months } \\
\text { of gaming/ } \\
\text { gamer }\end{array}$ & $\begin{array}{l}\text { Addicted } \\
\text { gamers }\end{array}$ & $\begin{array}{l}\text { Problematic } \\
\text { gamers }\end{array}$ & $\begin{array}{l}\text { Engaged } \\
\text { gamers }\end{array}$ & $\begin{array}{c}\text { Nonproblematic } \\
\text { gamers }\end{array}$ \\
\hline $\begin{array}{l}\text { South } \\
\text { Africa }\end{array}$ & 2580 & $21 \pm 3$ & $\begin{array}{c}2194 \\
(85.04 \%)\end{array}$ & $\begin{array}{c}386 \\
(14.96 \%) \\
\end{array}$ & $17 \pm 2.5$ & $14 \pm 2$ & $\begin{array}{c}820 \\
(31.78 \%)\end{array}$ & $\begin{array}{c}805 \\
(31.2 \%)\end{array}$ & $\begin{array}{c}105 \\
(4.07 \%)\end{array}$ & $\begin{array}{c}850 \\
(32.95 \%)\end{array}$ \\
\hline Cameroon & 1456 & $19 \pm 3$ & $\begin{array}{c}1300 \\
(89.28 \%)\end{array}$ & $\begin{array}{c}156 \\
(10.72 \%)\end{array}$ & $10 \pm 1.75$ & $19 \pm 1$ & $\begin{array}{c}456 \\
(31.32 \%)\end{array}$ & $\begin{array}{c}435 \\
(29.87 \%) \\
\end{array}$ & $\begin{array}{c}85 \\
(5.84 \%) \\
\end{array}$ & $\begin{array}{c}480 \\
(32.97 \%)\end{array}$ \\
\hline Ivory Coast & 908 & $23 \pm 2$ & $\begin{array}{c}785 \\
(86.45 \%)\end{array}$ & $\begin{array}{c}123 \\
(13.55 \%)\end{array}$ & $12 \pm 1.25$ & $13 \pm 1$ & $\begin{array}{c}278 \\
(30.62 \%)\end{array}$ & $\begin{array}{c}227 \\
(25 \%)\end{array}$ & $\begin{array}{c}53 \\
(5.84 \%)\end{array}$ & $\begin{array}{c}350 \\
(38.54 \%)\end{array}$ \\
\hline Gabon & 449 & $20 \pm 2$ & $\begin{array}{c}414 \\
(92.2 \%)\end{array}$ & $\begin{array}{c}35 \\
(7.8 \%)\end{array}$ & $6 \pm 0.5$ & $15 \pm 1$ & $\begin{array}{c}134 \\
(29.84 \%)\end{array}$ & $\begin{array}{c}123 \\
(27.4 \%)\end{array}$ & $\begin{array}{c}46 \\
(10.24 \%)\end{array}$ & $\begin{array}{c}146 \\
(32.52 \%)\end{array}$ \\
\hline Morocco & 1831 & $20 \pm 2$ & $\begin{array}{c}1662 \\
(90.77 \%)\end{array}$ & $\begin{array}{c}169 \\
(9.23 \%)\end{array}$ & $18 \pm 2.5$ & $18 \pm 2$ & $\begin{array}{c}504 \\
(27.53 \%)\end{array}$ & $\begin{array}{c}497 \\
(27.14 \%)\end{array}$ & $\begin{array}{c}157 \\
(8.57 \%)\end{array}$ & $\begin{array}{c}673 \\
(36.76 \%)\end{array}$ \\
\hline Nigeria & 1298 & $25 \pm 2$ & $\begin{array}{c}1147 \\
(88.37 \%)\end{array}$ & $\begin{array}{c}151 \\
(11.63 \%)\end{array}$ & $12 \pm 2.25$ & $13 \pm 1.75$ & $\begin{array}{c}350 \\
(26.96 \%)\end{array}$ & $\begin{array}{c}350 \\
(26.96 \%)\end{array}$ & $\begin{array}{c}126 \\
(9.71 \%)\end{array}$ & $\begin{array}{c}472 \\
(36.37 \%)\end{array}$ \\
\hline Rwanda & 897 & $25 \pm 1$ & $\begin{array}{c}809 \\
(90.19 \%)\end{array}$ & $\begin{array}{c}88 \\
(9.81 \%)\end{array}$ & $8 \pm 2$ & $10 \pm 2$ & $\begin{array}{c}260 \\
(29 \%)\end{array}$ & $\begin{array}{c}230 \\
(25.64 \%)\end{array}$ & $\begin{array}{c}146 \\
(16.27 \%)\end{array}$ & $\begin{array}{c}261 \\
(29.09 \%)\end{array}$ \\
\hline Sénégal & 846 & $22 \pm 2$ & $\begin{array}{c}770 \\
(91 \%)\end{array}$ & $\begin{array}{c}76 \\
(9 \%)\end{array}$ & $6 \pm 2.5$ & $11 \pm 0.25$ & $\begin{array}{c}211 \\
(24.94 \%)\end{array}$ & $\begin{array}{c}320 \\
(37.83 \%)\end{array}$ & $\begin{array}{c}54 \\
(6.38 \%)\end{array}$ & $\begin{array}{c}261 \\
(30.85 \%)\end{array}$ \\
\hline Tunisia & 301 & $26 \pm 4$ & $\begin{array}{c}285 \\
(94.68 \%)\end{array}$ & $\begin{array}{c}16 \\
(5.32 \%)\end{array}$ & $20 \pm 2.5$ & $26 \pm 2$ & $\begin{array}{c}57 \\
(18.94 \%)\end{array}$ & $\begin{array}{c}83 \\
(27.58 \%)\end{array}$ & $\begin{array}{c}73 \\
(24.25 \%)\end{array}$ & $\begin{array}{c}88 \\
(29.23 \%)\end{array}$ \\
\hline
\end{tabular}

FIGURE 1: Descriptive epidemiology of gaming among the nine African countries where prevalence of gaming, mean hours of gaming per week, period from when participant considered himself a gamer, and type of device used for gaming purposes are described with age and sex [7].

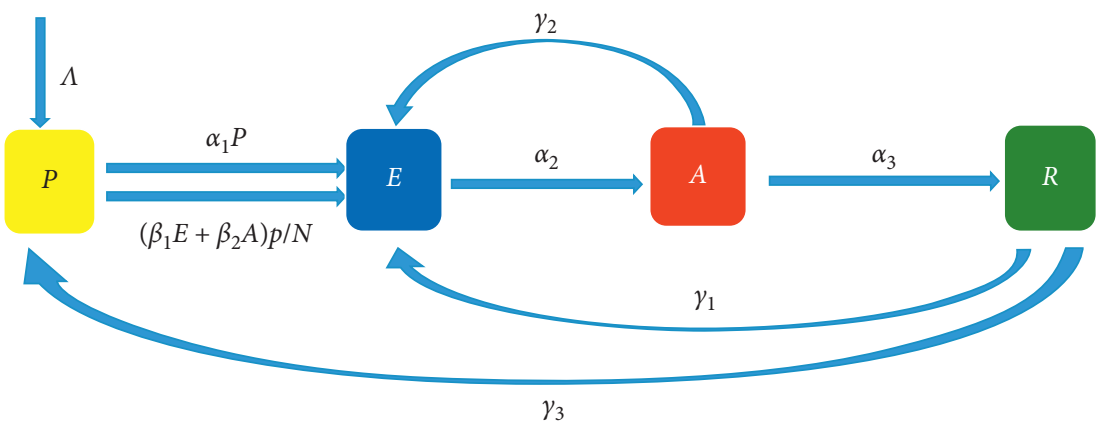

FiguRE 2: Illustration of movement between compartments.

other engaged gamers) and with addicted gamers at rate $\beta_{2}$ (the rate of patients who become engaged gamers because of the negative contact with the addicted gamers).

2.1.2. The Engaged Gamers E. It represents children and youth who are interested in electronic games and play more than four hours a day without secondary effects on the body and on the individual's behaviour in the social environment. This compartment is increased by $\alpha_{1}, \beta_{1}$, and $\beta_{2}$. Also, it is increased by $\gamma_{1}$ (the transmission factor from recovered persons to engaged gamers) and by $\gamma_{2}$ (the transmission factor from addicted gamers to engaged gamers). The compartment of engaged gamers is decreased by $\gamma_{2}$ that represents the rate of the engaged gamers who have become addicted gamers.
2.1.3. The Addicted Gamers A. It represents children and youth who are addicted to electronic games, suffer from gaming disorders, and have no control over their gaming habits. They prioritize gaming over other interests and activities and continue to game despite its negative consequences. This compartment is increased by $\alpha_{2}$ and it is decreased by $\gamma_{2}$. Also, it is decreased by the rate of the addicted gamers who have become recovered gamers denoted by $\gamma_{3}$.

2.1.4. The Recovered Gamers R. It represents children and youth recovering from their addiction to electronic games. This compartment is increased by $\alpha_{3}$. It is decreased by $\gamma_{3}$ and by the risk factor for transmission from recovered persons to engaged gamers $\gamma_{1}$. 
2.2. Model Equations. By adding the rates at which the steps of gaming disorder enter the compartment and also by subtracting the rates at which people leave a compartment, we obtain a system of difference equations for the rate at which patients change in each compartment during separate times. Therefore, we present the gaming disorder model with the following system of difference equations:

$$
\left\{\begin{array}{l}
P_{k+1}=\Lambda+\left(1-\alpha_{1}\right) P_{k}+\gamma_{3} R_{k}-\beta_{1} \frac{P_{k} E_{k}}{N}-\beta_{2} \frac{P_{k} A_{k}}{N}, \\
E_{k+1}=\alpha_{1} P_{k}+\left(1-\alpha_{2}\right) E_{k}+\gamma_{2} A_{k}+\gamma_{1} R_{k}+\beta_{1} \frac{P_{k} E_{k}}{N}+\beta_{2} \frac{P_{k} A_{k}}{N}, \\
A_{k+1}=\left(1-\alpha_{3}-\gamma_{2}\right) A_{k}+\alpha_{2} E_{k}, \\
R_{k+1}=\alpha_{3} A_{k}+\left(1-\gamma_{1}-\gamma_{3}\right) R_{k},
\end{array}\right.
$$

where $P_{0} \geq 0, E_{0} \geq 0, A_{0} \geq 0$, and $R_{0} \geq 0$ are given initial states. $\Lambda$ is the recruitment rate of potential gamers. $\alpha_{1}$ is coefficient of transmission from potential persons to engaged gamers due to the effects of advertising for electronic games through media. $\alpha_{2}$ is the rate of the engaged gamers who become addicted gamers. $\alpha_{3}$ is the rate of the addicted gamers who become recovered gamers. $\beta_{1}$ is the rate of patients who become engaged gamers because of the negative contact with the other engaged gamers. $\beta_{2}$ is the rate of patients who become engaged gamers because of the negative contact with the addicted gamers. $\gamma_{1}$ is the transmission factor from recovered persons to engaged gamers. $\gamma_{2}$ is the transmission factor from addicted gamers to engaged gamers. $\gamma_{3}$ is the risk factor for transmission from recovered persons to potential gamers.

In order to demonstrate the efficiency of the model, we propose a numerical simulation (see Figure 3 ) which allows us to see how the growth results adapt to reality to some extent. We calculated transactions $\alpha_{1}, \alpha_{2}, \beta_{1}$, and $\beta_{2}$ based on the statistical results for Morocco included in [7], and we used the Moroccan population census data (see $[7,21]$ ) to determine the $N_{0}, P_{0}, E_{0}, A_{0}$, and $\Lambda$.

Figure 3 shows that there is a rapid development of the number of the engaged gamers and on the other hand, there is a significant increase in the number of addicted gamers in a few months. It is clearly seen from Figure 3 that the phenomenon of gaming is in constant increase.

At present and in the light of the rapid development of computer technology and consequently the development and spread of electronic games, the world including Morocco has witnessed an increase in the number of addicts to electronic games. In fact, the addiction of children and

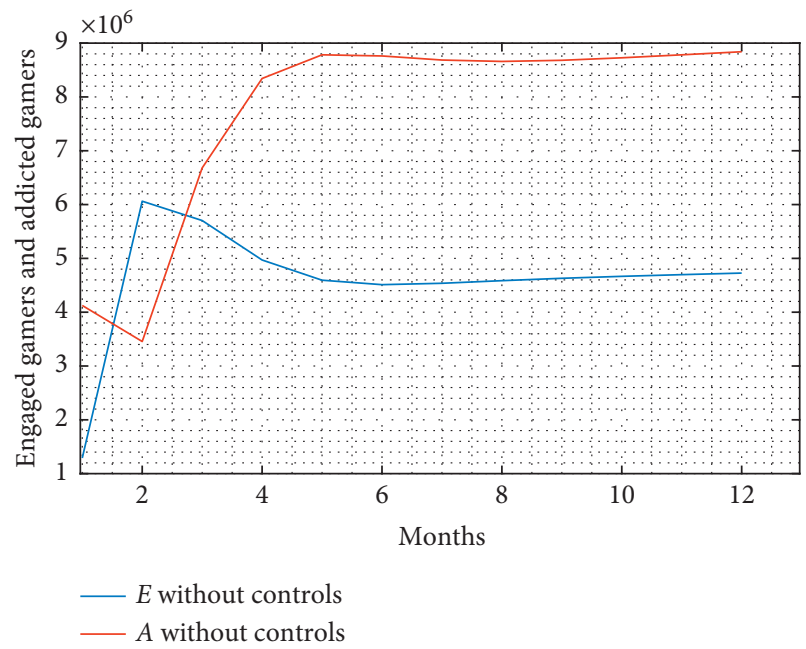

FIgURE 3: Development of the number of the engaged gamers and the addicted gamers in a few months.

young people leads to a sharp decline in the level of academic achievement, productivity at work and introspection, and a strong tendency to isolation. To address this phenomenon and mitigate its effects on individuals and society, we have proposed a set of practical strategies, which we present in the following paragraph.

\section{The Optimal Control Problem}

Our objective in the proposed control strategy is to minimize the number of engaged gamers and the number of addicted gamers. Therefore, in model (1), we include the controls: $u=\left(u_{0}, u_{1}, \ldots \ldots \ldots, u_{T-1}\right)$, which represents the effort to raise awareness of the dangers of electronic gaming and the dangers of addiction through written and visual media. Hence, the term $\left(1-u_{k}\right)$ is used to reduce the number of the affected individuals. In order to provide harmless alternatives for gaming which encompass educational games that help children and young people learn innovative programming skills, benefit themselves and society, and help them overcome their addiction to electronic games, we propose the control $v=\left(v_{0}, v_{1}, \ldots \ldots ., v_{T-1}\right)$ that represents the effort to provide these alternatives to children and young people to compensate for their addiction to electronic games. Some games reach a dangerous level of addiction which necessarily requires joining gaming rehabilitation centers that offer comprehensive treatment for such disorders. Hence, we propose the control $w=\left(w_{0}\right.$, $\left.w_{1}, \ldots \ldots \ldots, w_{T-1}\right)$ that represents the effort for creating rehabilitation center for addicts to quit electronic game addiction. 
Thus, the controlled mathematical system is given by the following system of difference equations:

$$
\left\{\begin{array}{l}
P_{k+1}=\Lambda+\left(1-\alpha_{1}\right) P_{k}+\gamma_{3} R_{k}-\left(1-u_{k}\right) P_{k}\left(\frac{\beta_{1} E_{k}}{N}+\frac{\beta_{2} A_{k}}{N}\right) \\
E_{k+1}=\alpha_{1} P_{k}+\left(1-\alpha_{2}\right) E_{k}+\left(\gamma_{2}+v_{k}\right) A_{k}+\gamma_{1} R_{k}+\left(1-u_{k}\right) P_{k}\left(\frac{\beta_{1} E_{k}}{N}+\frac{\beta_{2} A_{k}}{N}\right) \\
A_{k+1}=\left(1-\alpha_{3}-\gamma_{2}-w_{k}-v_{k}\right) A_{k}+\alpha_{2} E_{k} \\
R_{k+1}=\left(\alpha_{3}+w_{k}\right) A_{k}+\left(1-\gamma_{1}-\gamma_{3}\right) R_{k}
\end{array}\right.
$$

where $P_{0} \geq 0, E_{0} \geq 0, A_{0} \geq 0$, and $R_{0} \geq 0$ are given initial states.

Then, the problem is to minimize the objective functional:

$$
J(u, v, w)=E_{T}+A_{T}+\sum_{k=0}^{T-1}\left(E_{k}+A_{k}+\frac{M_{k} u_{k}^{2}}{2}+\frac{F_{k} v_{k}^{2}}{2}+\frac{G_{k} w_{k}^{2}}{2}\right)
$$

where the parameters $M_{k}>0, F_{k}>0$, and $G_{k}>0$ for $k \in 0,1,2, \ldots \ldots, T-1$ are the cost coefficients.

They are selected to weigh the relative importance of $u_{k}$, $v_{k}$, and $w_{k}$ at time $k$.

$T$ is the final time. In other words, we seek the optimal controls $u, v$, and $w$ such that

$$
J\left(u^{*}, v^{*}, w^{*}\right)=\min _{(u, v, w) \in U_{a d}} J(u, v, w),
$$

where $U_{a d}$ is the set of admissible controls defined by $U_{a d}=$ $\left\{(u, v, w): u=\left(u_{0}, u_{1}, \ldots, u_{T-1}\right), v=\left(v_{0}, v_{1}, \ldots, v_{T-1}\right)\right.$ and $w=\left(w_{0}, w_{1}, \ldots, w_{T-1}\right) / 0 \leqslant u_{k} \leqslant 1, \quad 0 \leqslant v_{k} \leqslant 1 \quad$ and $\quad 0 \leqslant w_{k} \leqslant 1$; $k \in\{0,1,2, \ldots, T-1\}\}$.

The sufficient condition for the existence of the optimal controls $u, v$, and $w$ for the problems (2) and (3) comes from Theorem 1.

Theorem 1. There exist the optimal controls $u^{*}, v^{*}$, and $w^{*}$ such that

$$
J\left(u^{*}, v^{*}, w^{*}\right)=\min _{(u, v, w) \in U_{a d}} J(u, v, w),
$$

subject to the control system (2) with initial conditions.

Proof. Since the coefficients of the state equations are bounded and there are a finite number of time steps,

$$
P=\left(P_{0}, P_{1}, \ldots ., P_{T}\right) ; E=\left(E_{0}, E_{1}, \ldots ., E_{T}\right) ; A=
$$

$\left(A_{0}, A_{1}, \ldots \ldots, A_{T}\right)$ and $R=\left(R_{0}, R_{1}, \ldots \ldots, R_{T}\right)$ are uniformly bounded for all $(u, v, w)$ in the controls set
$U_{a d}$ and thus $\mathrm{J}(\mathrm{u}, \mathrm{v}, \mathrm{w})$ is bounded for all $(u, v, w) \in U_{a d}$ since $J(u, v, w)$ is bounded.

$\inf _{(u, v, w) \in U_{a d}} J(u, v, w)$ is finite, and there exists a sequence $\left(u^{n}, v^{n}, w^{n}\right) \in U_{a d} \quad$ such that $\lim _{n \longrightarrow+\infty} J\left(u^{n}, v^{n}, w^{n}\right)=\inf _{(u, v, w) \in U_{a d}} J(u, v, w) \quad$ and corresponding sequences of states $P^{n}, E^{n}, A^{n}, R^{n}$; since there are a finite number of uniformly bounded sequences, there exist $\left(u^{*}, v^{*}, w^{*}\right) \in U_{a d}$ and $P^{*}, E^{*}, A^{*}$, and $R^{*} \in \mathbb{R}^{T+1} \quad$ such that sequences $u^{n} \longrightarrow u^{*}, v^{n} \longrightarrow v^{*}, w^{n} \longrightarrow w^{*}, P^{n} \longrightarrow P^{*}, E^{n} \longrightarrow$ $E^{*}, A^{n} \longrightarrow A^{*}$, and $R^{n} \longrightarrow R^{*}$.

Finally, due to the finite-dimensional structure of system (2) and the objective function $\mathrm{J}(\mathrm{u}, \mathrm{v}, \mathrm{w}), u^{*}, v^{*}$, and $w^{*}$ are the optimal controls with corresponding states $P^{*}, E^{*}, A^{*}, \quad$ and $R^{*}$. Therefore, $\inf _{(u, v, w) \in U_{a d}} J(u, v, w)$ is achieved.

In order to derive the necessary condition for the optimal controls, we use the discrete version of Pontryagin's maximum principle [22-30]. The idea is to introduce the adjoint function to attach the system of difference equations to the objective function resulting in the formation of a function called the Hamiltonian. This principle converts into a problem of minimizing à Hamiltonian $H_{k}$ at time step $k$ defined by

$$
H_{k}=E_{k}+A_{k}+\frac{M_{k} u_{k}^{2}}{2}+\frac{F_{k} v_{k}^{2}}{2}+\frac{G_{k} w_{k}^{2}}{2}+\sum_{i=1}^{4} \lambda_{i, k+1} f_{i, k+1},
$$

where $f_{i, k+1}$ is the right side of the system of difference equation (2) of the $i^{\text {th }}$ state variable at time step $k+1$. Using Pontryagin's maximum principle in discrete time [31-33], we state Theorem 2 .

Theorem 2. Given the optimal controls $u^{*}, v^{*}, w^{*}$, and the solutions $P^{*}, E^{*}, A^{*}$, and $R^{*}$ of the corresponding state system (2), there exist adjoint variables $\lambda_{1, k}, \lambda_{2, k}, \lambda_{3, k}$, and $\lambda_{4, k}$ satisfying 


$$
\left\{\begin{array}{l}
\lambda_{1, k}=\lambda_{1, k+1}\left(1-\alpha_{1}-\left(1-u_{k}\right)\left(\beta_{1} \frac{E_{k}}{N}+\beta_{2} \frac{A_{k}}{N}\right)\right)+\lambda_{2, k+1}\left(\alpha_{1}+\left(1-u_{k}\right)\left(\beta_{1} \frac{E_{k}}{N}+\beta_{2} \frac{A_{k}}{N}\right)\right) \\
\lambda_{2, k}=1-\lambda_{1, k+1} \beta_{1}\left(1-u_{k}\right) \frac{P_{k}}{N}+\lambda_{2, k+1}\left(1-\alpha_{2}+\beta_{1}\left(1-u_{k}\right) \frac{P_{k}}{N}\right)+\lambda_{3, k+1} \alpha_{2} \\
\lambda_{3, k}=1-\lambda_{1, k+1} \beta_{2}\left(1-u_{k}\right) \frac{P_{k}}{N}+\lambda_{2, k+1}\left(\gamma_{2}+v_{k}+\beta_{2}\left(1-u_{k}\right) \frac{P_{k}}{N}\right)+\lambda_{3, k+1}\left(1-\alpha_{3}-\gamma_{2}-v_{k}-w_{k}\right) \\
+\lambda_{4, k+1}\left(\alpha_{3}+w_{k}\right) \\
\lambda_{4, k}=\lambda_{1, k+1} \gamma_{3}+\lambda_{2, k+1} \gamma_{1}+\lambda_{4, k+1}\left(1-\gamma_{1}-\gamma_{3}\right)
\end{array}\right.
$$

With the transversality conditions at time $T, \lambda_{1, T}=0$; $\lambda_{2, T}=1 ; \lambda_{3, T}=1 ; \lambda_{4, T}=0$.

Furthermore, for $k=0,1,2, \ldots \ldots, T-1$, the optimal controls $u_{k}^{*}, v_{k}^{*}$, and $w_{k}^{*}$ are given by

$$
u_{k}^{*}=\min \left(u_{\max }, \max \left(u_{\min } \frac{\left(\lambda_{2, k+1}-\lambda_{1, k+1}\right) P_{k}\left(\beta_{1} E_{k}+\beta_{2} A_{k}\right)}{N M_{k}}\right)\right),
$$

$$
\begin{aligned}
& v_{k}^{*}=\min \left(v_{\max }, \max \left(v_{\min }, \frac{\left(\lambda_{3, k+1}-\lambda_{2, k+1}\right) A_{k}}{F_{k}}\right)\right), \\
& w_{k}^{*}=\min \left(w_{\max }, \max \left(w_{\min } \frac{\left(\lambda_{3, k+1}-\lambda_{4, k+1}\right) A_{k}}{G_{k}}\right)\right) .
\end{aligned}
$$

Proof. The Hamiltonian at time step $k$ is given by

$$
\begin{aligned}
H_{k}= & E_{k}+A_{k}+\frac{M_{k} u_{k}^{2}}{2}+\frac{F_{k} v_{k}^{2}}{2}+\frac{G_{k} w_{k}^{2}}{2}+\lambda_{1, k+1}\left(\Lambda+\left(1-\alpha_{1}\right) P_{k}+\gamma_{3} R_{k}-\left(1-u_{k}\right) P_{k}\left(\frac{\beta_{1} E_{k}}{N}+\frac{\beta_{2} A_{k}}{N}\right)\right) \\
& +\lambda_{2, k+1}\left(\alpha_{1} P_{k}+\left(1-\alpha_{2}\right) E_{k}+\left(\gamma_{2}+v_{k}\right) A_{k}+\gamma_{1} R_{k}+\left(1-u_{k}\right) P_{k}\left(\frac{\beta_{1} E_{k}}{N}+\frac{\beta_{2} A_{k}}{N}\right)\right) \\
& +\lambda_{3, k+1}\left(\left(1-\alpha_{3}-\gamma_{2}-v_{k}-w_{k}\right) A_{k}+\alpha_{2} E_{k}\right)+\lambda_{4, k+1}\left(\left(\alpha_{3}+w_{k}\right) A_{k}+\left(1-\gamma_{1}-\gamma_{3}\right) R_{k}\right),
\end{aligned}
$$

for $k=0,1,2, \ldots \ldots, T-1$, the adjoint equations and transversality conditions can be obtained by using
Pontryagin's maximum principle in discrete time given in [22-30] such that 


$$
\left\{\begin{array}{l}
\lambda_{1, k}=\frac{\mathrm{d} H_{k}}{\mathrm{~d} P_{k}}=\lambda_{1, k+1}\left(1-\alpha_{1}-\left(1-u_{k}\right)\left(\beta_{1} \frac{E_{k}}{N}+\beta_{2} \frac{A_{k}}{N}\right)\right)+\lambda_{2, k+1}\left(\alpha_{1}+\left(1-u_{k}\right)\left(\beta_{1} \frac{E_{k}}{N}+\beta_{2} \frac{A_{k}}{N}\right)\right), \\
\lambda_{2, k}=\frac{\mathrm{d} H_{k}}{\mathrm{~d} E_{k}}=1-\lambda_{1, k+1} \beta_{1}\left(1-u_{k}\right) \frac{P_{k}}{N}+\lambda_{2, k+1}\left(1-\alpha_{2}+\beta_{1}\left(1-u_{k}\right) \frac{P_{k}}{N}\right)+\lambda_{3, k+1} \alpha_{2}, \\
\lambda_{3, k}=\frac{\mathrm{d} H_{k}}{\mathrm{~d} A_{k}}=1-\lambda_{1, k+1} \beta_{2}\left(1-u_{k}\right) \frac{P_{k}}{N}+\lambda_{2, k+1}\left(\gamma_{2}+v_{k}+\beta_{2}\left(1-u_{k}\right) \frac{P_{k}}{N}\right) \\
+\lambda_{3, k+1}\left(1-\alpha_{3}-\gamma_{2}-v_{k}-w_{k}\right)+\lambda_{4, k+1}\left(\alpha_{3}+w_{k}\right), \\
\lambda_{4, k}=\frac{\mathrm{d} H_{k}}{\mathrm{~d} R_{k}}=\lambda_{1, k+1} \gamma_{3}+\lambda_{2, k+1} \gamma_{1}+\lambda_{4, k+1}\left(1-\gamma_{1}-\gamma_{3}\right),
\end{array}\right.
$$

and with the transversality conditions at time $T, \lambda_{1, T}=0$; $\lambda_{2, T}=1 ; \lambda_{3, T}=1 ; \lambda_{4, T}=0$. For $k \in 0,1,2, \ldots \ldots, T-1$, the optimal controls $u_{k}^{*}, v_{k}^{*}$, and $w_{k}^{*}$ can be solved from the optimality condition $\left(\mathrm{d} H_{k} / \mathrm{d} u_{k}\right)=0,\left(\mathrm{~d} H_{k} / \mathrm{d} v_{k}\right)=0$, and $\left(\mathrm{d} H_{k} / \mathrm{d} w_{k}\right)=0$; that is,

$$
\begin{aligned}
& \frac{\mathrm{d} H_{k}}{\mathrm{~d} u_{k}}=M_{k} u_{k}+\left(\lambda_{1, k+1}-\lambda_{2, k+1}\right)\left(\beta_{1} E_{k}+\beta_{2} A_{k}\right) \frac{P_{k}}{N}=0, \\
& \frac{\mathrm{d} H_{k}}{\mathrm{~d} v_{k}}=F_{k} v_{k}+\lambda_{2, k+1} A_{k}-\lambda_{3, k+1} A_{k}=0, \\
& \frac{\mathrm{d} H_{k}}{\mathrm{~d} w_{k}}=G_{k} w_{k}-\lambda_{3, k+1} A_{k}-\lambda_{4, k+1} A_{k}=0,
\end{aligned}
$$

so we have

$$
\begin{aligned}
& u_{k}=\frac{\left(\lambda_{2, k+1}-\lambda_{1, k+1}\right) P_{k}\left(\beta_{1} E_{k}+\beta_{2} A_{k}\right)}{N M_{k}}, \\
& v_{k}=\frac{\left(\lambda_{3, k+1}-\lambda_{2, k+1}\right) A_{k}}{F_{k}}, \\
& w_{k}=\frac{\left(\lambda_{3, k+1}-\lambda_{4, k+1}\right) A_{k}}{G_{k}},
\end{aligned}
$$

and by the bounds in $U_{a d}$ of the controls, it is easy to obtain $u_{k}^{*}, v_{k}^{*}$, and $w_{k}^{*}$ in the form (8)-(10).

\section{Numerical Simulation and Cost- Effectiveness Analysis}

In this section, we present the results obtained by numerically solving the optimality system. As explained in [14-20], the optimality system is a two-point limit value problem system with separate boundary conditions at the times when the step $k=0$ and $k=T$. We solve the optimality system by an iterative method with forward solving of the state system followed by backward solving of the adjoint system. We start with an initial guess for the controls at the first iteration and then before the next iteration, we update the controls by using the characterization. We continue until convergence of successive iterates is achieved. A code is written and compiled in MATLAB; we use the data in Table 1.To obtain results that simulate reality to some extent, we calculated transactions $\alpha_{1}, \alpha_{2}, \beta_{1}$, and $\beta_{2}$ based on the statistical results for Morocco included in [7], and we used the Moroccan population census data (see $[7,21]$ ) to determine the $N_{0}, P_{0}, E_{0}, A_{0}$, and $\Lambda$.

4.1. Discussion. In this section, we offer a digital simulation to highlight the effectiveness of our strategy to combat the impact of addiction to electronic games on children and young people and reduce their addiction. Different simulations can be carried out using various values of parameters as shown in Table 1. We introduce our control strategy which consists of using three types of controls: the first one is symbolized by $u_{k}$ and represents awareness of the dangers of electronic games through written and visual media, the second one is symbolized by $v_{k}$ and represents the effort to directing children and adolescents to educational and entertaining alternative means, and the third one is symbolized by $w_{k}$ and represents the effort for creating rehabilitation center for addicts to quit electronic game addiction. Furthermore, we investigate numerically the impact of each of the following optimal control strategies.

Scenario 1. In this scenario, we simulate the case where we apply a single control $u_{k}$, raising awareness of the dangers of electronic games through written and visual media. In Figure 4, we see that, after 12 months, the number of addicted gamers decreased slightly from $8.8 * 10^{6}$ (without control) to $8.3 * 10^{6}$ (with control). Also, for the number of engaged gamers as shown in Figure 5, it decreased slightly from $4.7 * 10^{6}$ (without control) to $4.5 * 10^{6}$ (with control).

Scenario 2. In this scenario, applying the control $v_{k}$ which represents providing harmless alternatives as means for education and entertainment for children and adolescents, we see that, after 12 months as shown in Figure 4 , the number of addicted gamers decreased slightly from $8.8 * 10^{6}$ (without control) to $8.2 * 10^{6}$ (with control). In Figure 5, the 
TABle 1: The description of the parameters data used for systems (1).

\begin{tabular}{|c|c|c|c|}
\hline Parameter & Description & Estimated value & Source \\
\hline$N_{0}$ & The number of children and youth in Morocco & 15000000 & [21] \\
\hline$P_{0}$ & Initial number of potential gamers & 9585000 & [7] \\
\hline$E_{0}$ & Initial number of engaged gamers & 1290000 & [7] \\
\hline$A_{0}$ & Initial number of addicted gamers & 4125000 & [7] \\
\hline$R_{0}$ & Initial number of recovered gamers & 0 & Assumed \\
\hline$\Lambda$ & The incidence of potential gamers & 7988 & {$[21]$} \\
\hline$\alpha_{1}$ & The rate of the potential gamers who become engaged gamers & 0.36 & [7] \\
\hline$\alpha_{2}$ & The rate of the engaged gamers who become addicted gamers & 0.76 & [7] \\
\hline$\alpha_{3}$ & The rate of the addicted gamers who become recovered gamers & 0.1 & Assumed \\
\hline$\beta_{1}$ & The rate of the $P$ who become engaged gamers by contact with engaged gamers & 0.46 & [7] \\
\hline$\beta_{2}$ & The rate of the $\mathrm{P}$ who become engaged gamers by contact with addicted gamers & 0.26 & [7] \\
\hline$\gamma_{1}$ & The rate of the recovered gamers who become engaged gamers & 0.2 & Assumed \\
\hline$\gamma_{2}$ & The rate of the addicted gamers who become engaged gamers & 0.1 & Assumed \\
\hline$\gamma_{3}$ & The rate of the recovered gamers who become potential gamers & 0.4 & Assumed \\
\hline
\end{tabular}

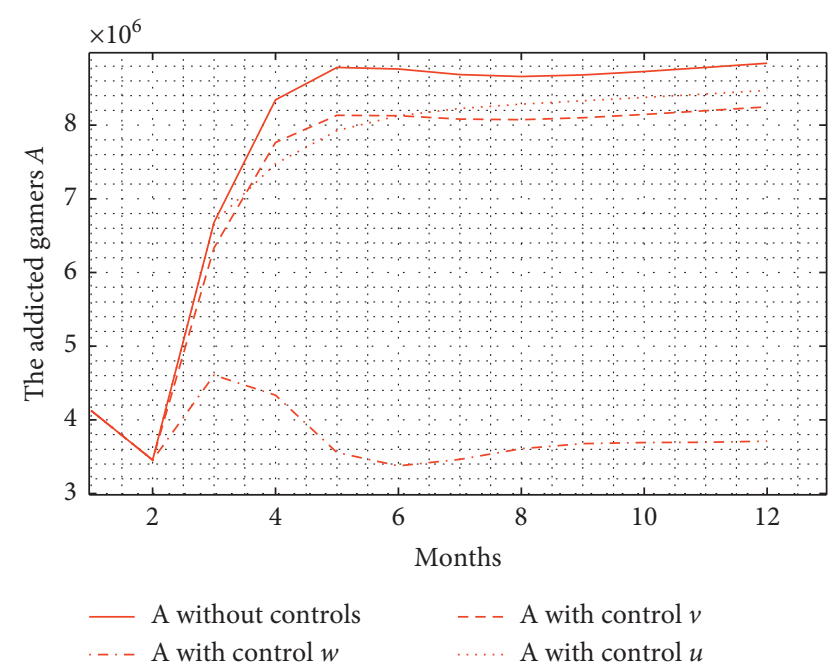

FIGURE 4: Development of the number of the addicted gamers with one control in a few months.

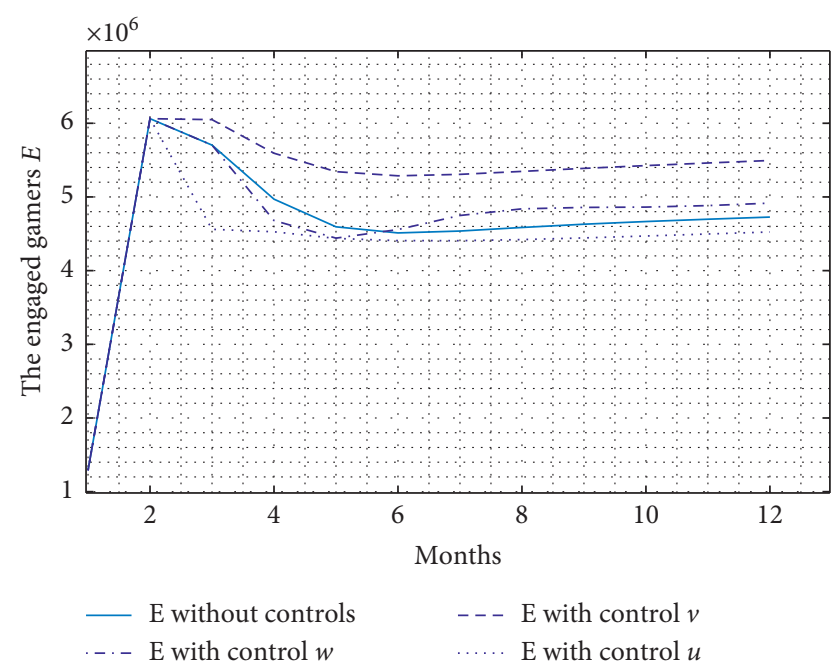

FIGURE 5: Development of the number of the engaged gamers with one control in a few months. number of engaged gamers increased from $4.7 * 10^{6}$ (without control) to $5.5 * 10^{6}$ (with control).

Scenario 3. In this scenario, we apply the control $w_{k}$, creating gaming rehabilitation centers for the addicts to electronic games. We see that, after 12 months as shown in Figure 4 , the number of addicted gamers decreased from $8.8 * 10^{6}$ (without control) to $3.7 * 10^{6}$ (with control), which demonstrates the effectiveness of this intervention in reducing the number of the addicted gamers. In Figure 5, the number of the engaged gamers increased from $4.7 * 10^{6}$ (without control) to $4.9 * 10^{6}$ (with control).

Scenario 4. In this scenario, we combine Scenarios 1 and 2. We see that, after 12 months as shown in Figure 6, the number of the addicted gamers decreased from $8.8 * 10^{6}$ (without control) to $7.9 * 10^{6}$ (with controls). In Figure 7, the number of the engaged gamers increased from $4.7 * 10^{6}$ (without control) to $5.25 * 10^{6}$ (with controls).

Scenario 5. In this scenario, we combine Scenarios 1 and 3 by using the controls $u_{k}$ and $w_{k}$. We see that, after 12 months, as shown in Figure 6, the number of the addicted gamers decreased from $8.8 * 10^{6}$ (without control) to $3.4 * 10^{6}$ (with controls). In Figure 7 , the number of the engaged gamers decreased from $4.7 * 10^{6}$ (without control) to $4.42 * 10^{6}$ (with controls), which shows the effectiveness of the combination of these controls.

Scenario. 6In this scenario, we combine Scenario 2 and Scenario 3 by using the controls $v_{k}$ and $w_{k}$. We see that, after 12 months, as shown in Figure 6, the number of the addicted gamers decreased from $8.8 * 10^{6}$ (without control) to $3.6 * 10^{5}$ (with controls). In Figure 7 , the number of the engaged gamers increased from $4.7 * 10^{6}$ (without control) to $5.25 * 10^{6}$ (with controls).

Scenario 7. In this scenario, we combine all the three controls $u_{k}, v_{k}$, and $w_{k}$. We see that, after 12 months, as shown in Figure 8, the number of the addicted gamers decreased from $8.8 * 10^{6}$ (without controls) to $3.25 * 10^{6}$ 


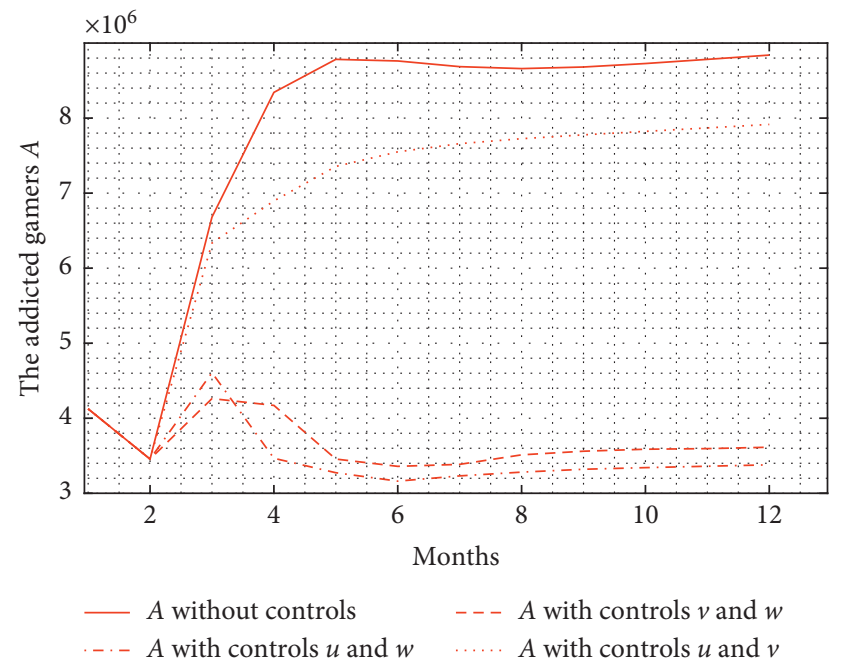

FIGURE 6: Development of the number of the addicted gamers with the combination of two controls among the controls $u_{k}, v_{k}$, and $w_{k}$.

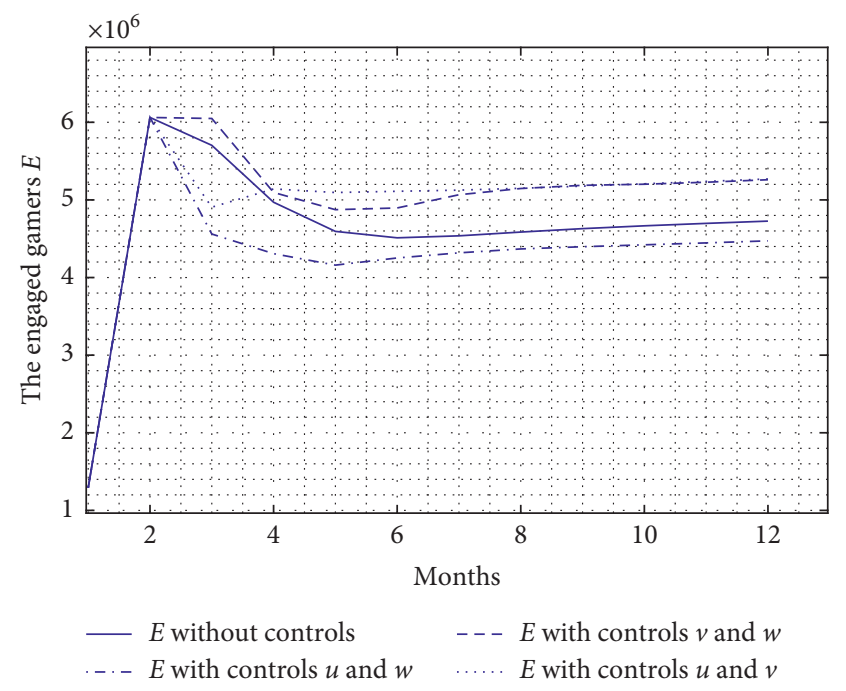

FIGURE 7: Development of the number of the engaged gamers with the combination of two controls among the controls $u_{k}, v_{k}$, and $w_{k}$.

(with controls), demonstrating the effectiveness of this intervention in reducing the number of the addicted gamers. In Figure 9, the number of engaged gamers increased slightly from $4.7 * 10^{6}$ (without controls) to $4.8 * 10^{6}$ (with controls).

Finally, in Figures 10(a)-10(c), we present the optimal control variables $u_{k}^{*}, v_{k}^{*}$, and $w_{k}^{*}$ used in the previous scenarios.

4.2. Cost-Effectiveness Analysis. In this section, we analyze the cost-effectiveness ratio (ICER) of the previous seven scenarios by comparing them to determine the most costeffectiveness ratio (ICER). Following the method as applied in several studies [34-36], this ratio was used to compare the differences between the costs and health outcomes of two

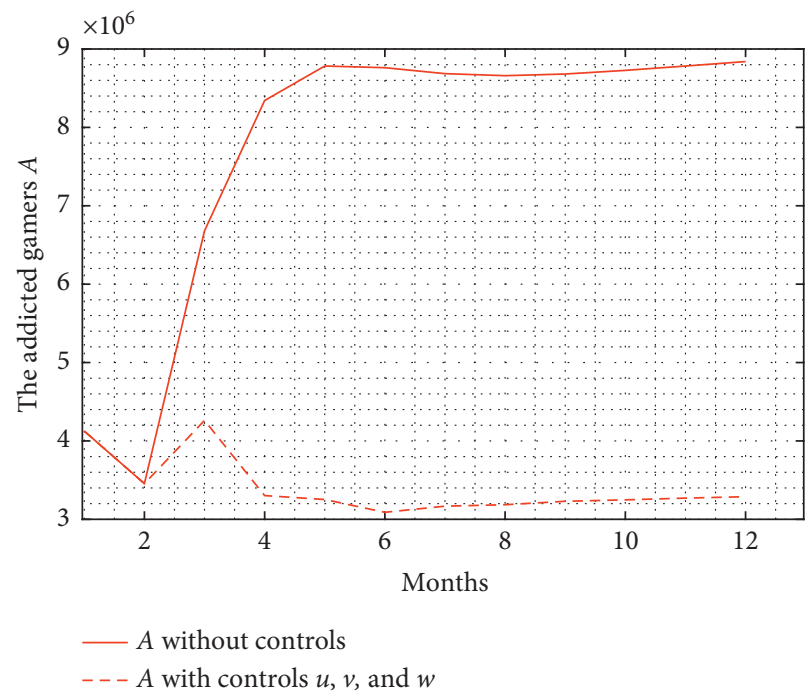

FIGURE 8: The evolution of the number of the addicted gamers with combination of the three controls.

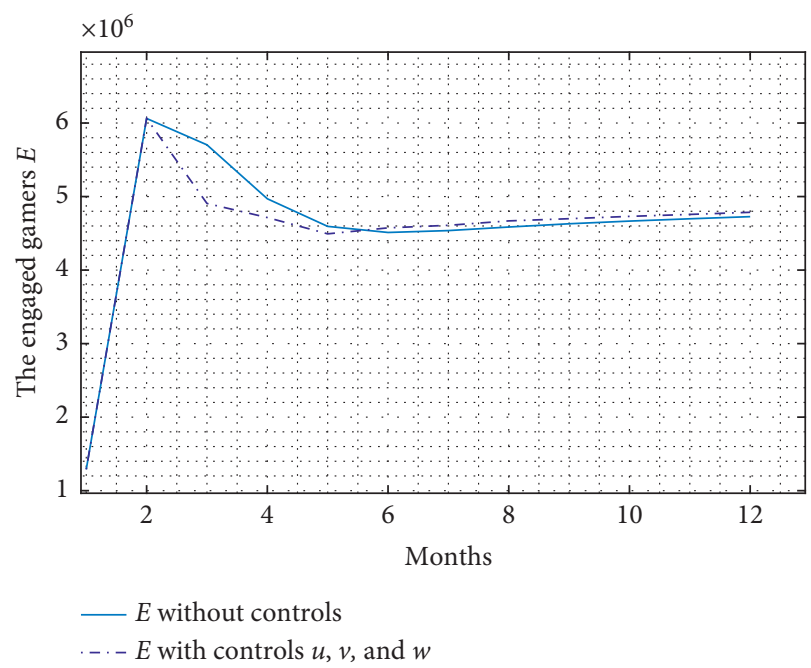

Figure 9: The evolution of the number of the engaged gamers with combination of the three controls.

competing strategies. The ICER is defined as the quotient of the difference in costs in strategies $i$ and $j$, by the difference in infected averted in strategies $i$ and $j(i, j \in\{1,2,3,4\})$. Given two competing strategies $i$ and $j$, where strategy $j$ has higher effectiveness than strategy $i(T A(i)<T A(j))$, the ICER values are calculated as follows:

$$
\begin{aligned}
\operatorname{ICER}(i) & =\frac{\operatorname{TC}(i)}{\operatorname{TA}(i)}, \\
\operatorname{ICER}(j) & =\frac{\operatorname{TC}(j)-\operatorname{TC}(i)}{\operatorname{TA}(j)-\operatorname{TA}(i)},
\end{aligned}
$$

where the total costs (TC) and the total cases averted (TA) are defined, in our study, during a given period for scenario $i$ for $i=1,2,3,4,5,6,7$ by 


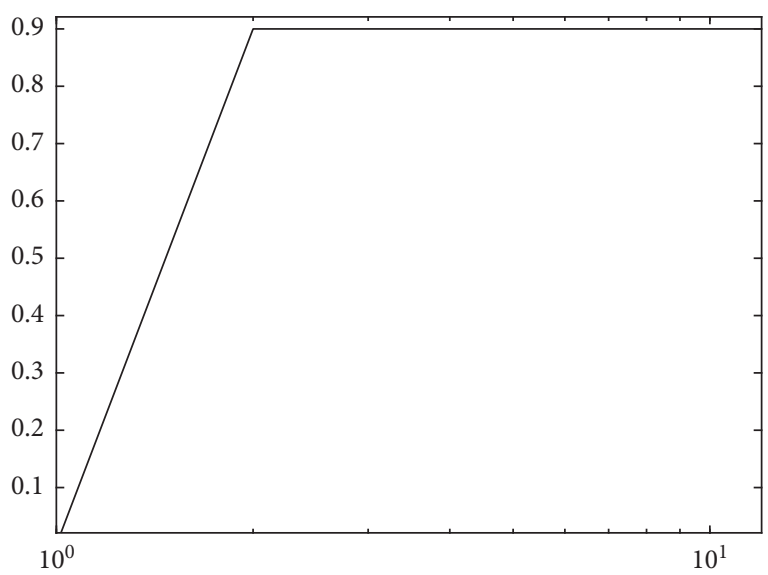

(a)

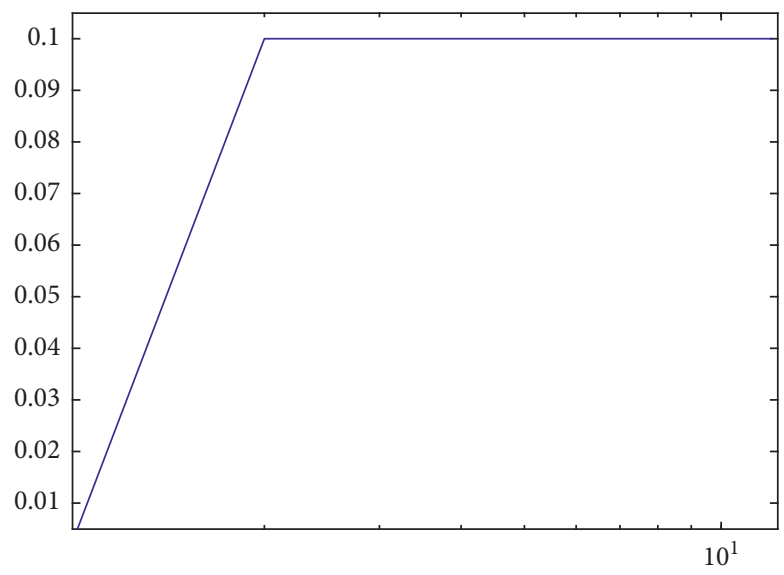

(b)

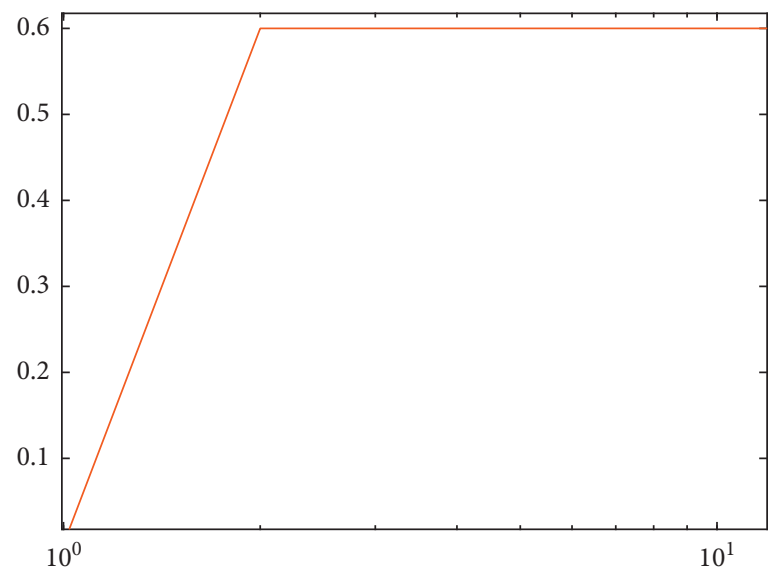

(c)

FIgURE 10: The evolution of all controls $u_{k}, v_{k}$, and $w_{k}$. (a) The optimal control $u_{k}$. (b) The optimal control $v_{k}$. (c) The optimal control $w_{k}$.

$$
\begin{aligned}
\mathrm{TC}(i)= & \sum_{k=0}^{T-1}\left(M_{k} u_{k}^{*} P_{k}^{*}+\left(F_{k} v_{k}^{*}+G_{k} w_{k}^{*}\right) A_{k}^{*}\right), \\
& \operatorname{TA}(i) \sum_{k=0}^{T}\left(\left(A_{k}+E_{k}\right)-\left(A_{k}^{*}+E_{k}^{*}\right)\right),
\end{aligned}
$$

where $M_{k}, F_{k}$, and $G_{k}$ correspond to the person unit cost of the three possible interventions, while $A_{k}^{*}$ and $E_{k}^{*}$ are the optimal solution associated to the optimal controls $u_{k}^{*}, v_{k}^{*}$, and $w_{k}^{*}$. Based on the model simulation results, we ranked in Table 2 our control strategies in order of increased numbers of averted infections.

First, we compared the cost-effectiveness of Scenarios 2 and 4:

$$
\begin{aligned}
& \operatorname{ICER}(2)=\frac{7.9205 * 10^{6}}{-0.0134 * 10^{8}}=-5.91 \\
& \operatorname{ICER}(4)=\frac{2.437 * 10^{7}-7.9205 * 10^{7}}{0.0683 * 10^{8}+0.0134 * 10^{8}}=-67.11 .
\end{aligned}
$$

We note that ICER (2) is higher than ICER (4). This means that Scenario 2 is dominated by Scenario 4 . Therefore, strategy 2 is excluded from the set of alternatives.
TABLE 2: Total costs and total averted infections for all scenarios.

\begin{tabular}{lcc}
\hline Strategy & Total averted infections (TA) & Total cost (TC) \\
\hline 2 & $-0.0134 * 10^{8}$ & $7.9205 * 10^{7}$ \\
4 & $0.0683 * 10^{8}$ & $2.437 * 10^{7}$ \\
1 & $0.0755 * 10^{8}$ & $1.739 * 10^{7}$ \\
6 & $0.4405 * 10^{8}$ & $2.5551 * 10^{7}$ \\
3 & $0.4635 * 10^{8}$ & $2.2633 * 10^{7}$ \\
7 & $0.5233 * 10^{8}$ & $5.8598 * 10^{7}$ \\
5 & $0.5444 * 10^{8}$ & $5.6818 * 10^{7}$ \\
\hline
\end{tabular}

Second, we compared the cost-effectiveness of Scenarios 4 and 1:

$$
\begin{aligned}
& \operatorname{ICER}(4)=\frac{2.437 * 10^{7}}{0.0683 * 10^{8}}=3.568 \\
& \operatorname{ICER}(1)=\frac{1.739 * 10^{7}-2.437 * 10^{7}}{0.0755 * 10^{8}-0.0683 * 10^{8}}=-9.694 .
\end{aligned}
$$

By comparing Scenarios 1 and 4, the lower ICER for Scenario 1 indicates that Scenario 4 is strongly dominated by Scenario 1. That is, Scenario 4 is more costly and less effective than Scenario 1. Therefore, Scenario 4 is excluded from the set of alternatives. 
Next, Scenario 1 is compared with Scenario 6:

$$
\begin{aligned}
& \operatorname{ICER}(1)=\frac{1.739 * 10^{7}}{0.0755 * 10^{8}}=2.3, \\
& \operatorname{ICER}(6)=\frac{2.5551 * 10^{7}-1.739 * 10^{7}}{0.4405 * 10^{8}-0.0755 * 10^{8}}=0.22 .
\end{aligned}
$$

Since $\operatorname{ICER}(6)<\operatorname{ICER}(1)$, then Scenario 1 is less effective than Scenario 6. Therefore, Scenario 1 is excluded from the set of alternatives.

Next, we compare the cost-effectiveness of Scenario 6 and Scenario 3:

$$
\begin{aligned}
& \operatorname{ICER}(6)=\frac{2.5551 * 10^{7}}{0.4405 * 10^{8}}=0.58 \\
& \operatorname{ICER}(3)=\frac{2.2633 * 10^{7}-2.5551 * 10^{7}}{0.4635 * 10^{8}-0.4405 * 10^{8}}=-1.26 .
\end{aligned}
$$

The comparison of ICER (3) and ICER (6) indicates that Scenario 6 is more costly than Scenario 3; this means that Scenario 6 is more expensive and less effective than Scenario 3. Therefore, Scenario 1 is excluded from the set of alternatives.

Now, we compare the cost-effectiveness of Scenario 3 and Scenario 7:

$$
\begin{aligned}
& \operatorname{ICER}(3)=\frac{2.2633 * 10^{7}}{0.4635 * 10^{8}}=0.488 \\
& \operatorname{ICER}(7)=\frac{5.8598 * 10^{7}-2.2633 * 10^{7}}{0.5233 * 10^{8}-0.4635 * 10^{8}}=6.01 .
\end{aligned}
$$

The lower ICER obtained for Scenario 3 is an indication that Scenario 3 strongly dominates Scenario 7 and this simply indicates that Scenario 7 is more costly to implement in comparison to Scenario 3. Therefore, it is best to exclude Scenario 7 from the set of control strategies and alternative interventions to implement in order to preserve limited resources.

Finally, Scenario 3 is compared with Scenario 5:

$$
\begin{aligned}
& \operatorname{ICER}(3)=\frac{2.2633 * 10^{7}}{0.4635 * 10^{8}}=0.488 \\
& \operatorname{ICER}(5)=\frac{5.6818 * 10^{7}-2.2633 * 10^{7}}{0.5444 * 10^{8}-0.4635 * 10^{8}}=4.225 .
\end{aligned}
$$

The comparison reveals that Scenario 3 is less costly than Scenario 5 by saving 3.737; therefore, Scenario 3 is the best strategy among all the compared strategies due to its costeffectiveness.

\section{Conclusion}

In this research, we proposed a new mathematical model that describes the dynamics of gaming disorder. The model will allow us to understand the phenomenon of the spread of this disorder and to deal with it. Thus, we applied three control strategies. As a result, we were able to study and combine several scenarios in order to see the impact and effect of each of these controls on the spread of this disorder. To obtain results that relatively simulate reality, we calculated transactions $\alpha_{1}, \alpha_{2}, \beta_{1}$, and $\beta_{2}$ based on the statistical results from Morocco included in [7], and we used the Moroccan population census data (see $[7,21]$ ) to determine $N_{0}, P_{0}, E_{0}, A_{0}$, and $\Lambda$. The numerical resolution of the system with the difference equations as well as the numerical simulation allowed us to compare and see the difference between each scenario in a concrete way. The numerical results prove the effectiveness of our strategy and its importance in the fight against the spread of gaming disorders in society, particularly among children and young people. Using ICER cost-effectiveness analysis, we showed that Scenario 3 is the most effective strategy. In the next research in this area, we will try to shed light on the social and economic causes and conditions that contribute to the spread of e-game addiction and to rely in this study on realistic statistics related to proliferation coefficients as well as the use of mathematical model using Atangana-Baleanu-Caputo fractional derivative.

\section{Data Availability}

No data were used to support this study.

\section{Conflicts of Interest}

The authors declare that they have no conflicts of interest.

\section{References}

[1] D. J. Kuss, M. D. Griffiths, and H. M. Pontes, "Chaos and confusion in DSM-5 diagnosis of Internet Gaming Disorder: issues, concerns, and recommendations for clarity in the field," Journal of Behavioral Addictions, vol. 6, no. 2, pp. 103-109, 2017.

[2] D. A. G. Pathological, "Video-game use among youth ages 8 to 18: a national study," Psychological Science, vol. 20, no. 5, pp. 594-602, 2009.

[3] P. K. Bender and D. A. Gentile, "Internet gaming disorder: relations between needs satisfaction in-game and in life in general," Psychology of Popular Media, vol. 9, no. 2, pp. 266-278, 2020.

[4] World Health Organization, "Sharpening the focus on gaming disorder," Bulletin of the World Health Organization, vol. 97, pp. 382-383, 2019.

[5] J. B. Funk, D. D. Buchman, and J. N. Germarm, "preference for violent electronic games, self concept, and gender differences in young children," American Journal of Orlhopsychiaty, vol. 70, no. 2, 2000.

[6] J. B. Funk, J. Hagan, J. Schimming, W. A. Bullock, D. D. Buchman, and M. Myers, "Aggression and psychopathology in adolescents with a preference for violent electronic games," Aggressive Behavior, vol. 28, no. 2, pp. 134-144, 2002.

[7] F. A. Etindele Sosso, D. J. Kuss, C. Vandelanotte et al., "Insomnia, sleepiness, anxiety and depression among different types of gamers in African countries," Scientific Reports, vol. 10, 2020.

[8] J. G. Brida and G. Cayssials, "A dynamic extension of the classical model of production prices determination," Journal of Dynamics and Games, vol. 7, 2020. 
[9] Z. Wang and O. Petrosian, "On class of non transferable utility cooperative differential games with continuous updating," Journal of Dynamics and Games, vol. 7, 2020.

[10] Y. Guo and T. Li, "Optimal control strategies for an online game addiction model with low and high risk exposure," Discrete and Continuous Dynamical Systems-B, vol. 26, 2021.

[11] J. O. Akanni, F. O. Akinpelu, S. Olaniyi, A. T. Oladipo, and A. W. Ogunsola, "Modelling financial crime population dynamics: optimal control and cost-effectiveness analysis," International Journal of Dynamics and Control, vol. 8, 2020.

[12] A. Kouidere, D. Kada, O. Balatif, M. Rachik, and M. Naim, "Optimal control approach of a mathematical modeling with multiple delays of the negative impact of delays in applying preventive precautions against the spread of the COVID-19 pandemic with a case study of Brazil and cost-effectiveness," Solitons and Fractals, vol. 142, Article ID 110438, 2021.

[13] M. A. Aba Oud, A. Ali, H. Alrabaiah, S. Ullah, M. Altaf Khan, and S. Islam, "A fractional order mathematical model for COVID-19 dynamics with quarantine, isolation, and environmental viral load," Advances in Difference Equations, vol. 2021, 2021.

[14] D. Kada, A. Kouidere, O. Balatif, M. Rachik, and E. H. Labriji, "Mathematical modeling of the spread of COVID-19 among different age groups in Morocco: optimal control approach for intervention strategies," Chaos, Solitons \& Fractals, vol. 141, Article ID 110437, 2020.

[15] D. Kada, A. Labzai, O. Balatif, M. Rachik, and H. Labriji, "Spread of COVID-19 in Morocco discrete mathematical modeling: optimal control strategies and cost-effectiveness analysis," Journal of Mathematical And Computational Science, vol. 10, no. 5, pp. 2070-2093, 2020.

[16] B. Khajji, D. Kada, O. Balatif, and M. Rachik, "Amulti-region discrete time mathematical modeling of the dynamics of COVID-19 virus propagation using optimal control," Journal of Applied Mathematics and Computing, vol. 64, 2020.

[17] Y. Guo and T. Li, "Optimal control and stability analysis of an online game addiction model with two stages," Mathematical Methods in the Applied Sciences, vol. 43, pp. 4391-4408, 2020.

[18] T. Li and Y. Guo, "Stability and optimal control in a mathematical model of online game addiction," Filomat, vol. 33, 2019.

[19] O. Balatif, A. Labzai, and M. Rachik, "A discrete mathematica modeling and optimal control of the electoral behavior with regard to a political party," Discrete Dynamics in Nature and Society, vol. 2018, Article ID 9649014, 14 pages, 2018.

[20] J. Karrakchou, M. Rachik, and S. Gourari, "Optimal control and infectiology: application to an HIV/AIDS model," Applied Mathematics and Computation, vol. 177, no. 2, pp. 807-818, 2006.

[21] https://www.hcp.ma/Population-par-groupes-d-agesfonctionnels-1960-2050_a681.html.

[22] J. Workman and S. Lenhart, Optimal Control Applied to Biological Models, Chapmal Hall/CRC, Boca Raton, FL, USA, 2007.

[23] W. O. Kermack and A. G. McKendrick, "A contribution to the mathematical theory of epidemics," Proceedings of the Royal Society of London. Series A, Containing Papers of a Mathematical and Physical Character, vol. 115, no. 772, pp. 700-721, 1927.

[24] D. C. Zhang and B. Shi, "Oscillation and global asymptotic stability in a discrete epidemic model," Journal of Mathematical Analysis and Applications, vol. 278, no. 1, pp. 194-202, 2003.
[25] Z. Hu, Z. Teng, and H. Jiang, "Stability analysis in a class of discrete SIRS epidemic models," Nonlinear Analysis: Real World Applications, vol. 13, no. 5, pp. 2017-2033, 2012.

[26] M. D. Rafal and W. F. Stevens, "Discrete dynamic optimization applied to on-line optimal control," AIChE Journal, vol. 14, no. 1, pp. 85-91, 1968.

[27] L. L. Cui, H. G. Zhang, D. Liu, and Y. S. Kim, "Constrained optimal control of affine nonlinear discrete-time systems using ghjb method," in Proceedings of the 2009 IEEE International Symposium on Adaptive Dynamic Programming and Reinforcement Learning, ADPRL, pp. 16-21, Nashville, TN, USA, April 2009.

[28] D. Liu, D. Wang, D. Zhao, Q. Wei, and N. Jin, "Neuralnetworkbased optimal control for a class of unknown discrete-time nonlinear systems using globalized dua heuristic programming," IEEE Transactions on Automation Science and Engineering, vol. 9, 2012.

[29] A. Kouidere, B. Khajji, A. El bhih, O. Balatif, and M. Rachik, "A mathematical modeling with optimal control strategy of transmission of COVID-19 pandemic virus," Communications in Mathematical Biology and Neuroscience, vol. 2020, 2020.

[30] A. Labzai, O. Balatif, and M. Rachik, “Optimal control strategy for a discrete time smoking model with specific saturated incidence rate," Discrete Dynamics in Nature and Society, vol. 2018, Article ID 5949303, 10 pages, 2018.

[31] L. S. Pontryagin, V. G. Boltyanskii, R. V. Gamkrelidze, and E. F. Mishchenko, The Mathematical Theory of Optimal Processes, Wiley, New York, NY, USA, 1962.

[32] V. Guibout and A. M. Bloch, "A discrete maximum principle for solving optimal control problems," in Proceedings of the 2004 43rd IEEE Conference on Decision and Control (CDC), pp. 1806-1811, IEEE, Nassau, Bahamas, December 2004.

[33] W. Ding, R. Hendon, B. Cathey, E. Lancaster, and R. Germick, "Discrete time optimal control applied to pest control problems," Involve, vol. 7, no. 4, pp. 479-489, 2014.

[34] K. O. Okosun, O. Rachid, and N. Marcus, "Optimal control strategies and cost-effectiveness analysis of a malaria model," Biosystems, vol. 111, no. 2, pp. 83-101, 2013.

[35] F. B. Agusto and I. M. ELmojtaba, "Optimal control and costeffective analysis of malaria/visceral leishmaniasis co-infection," PLoS One, vol. 12, no. 2, Article ID e0171102, 2017.

[36] N. Hidayat and R. B. E. Wibowo, "Optimal control and costeffectiveness analysis of HIV model with educational campaigns and therapy," Matematika: MJIAM, vol. 35, 2019. 